In den Ausdrücken für $\frac{x_{1}^{\prime}}{r_{1}{ }^{3}}, \frac{y_{1}^{\prime}}{r_{1}^{3}}, \frac{z_{1}{ }^{\prime}}{r_{1}^{3}}$ treten daher noch die Glieder auf

wobei

$$
\begin{gathered}
\left(r_{1}\right) \cos \left(v_{1}+\Pi\right) \cdot{ }_{i=-\infty} \sum_{i=-\infty}^{+\infty} Y^{(i)} \cos i M_{1}=1 / 2 \sum_{i=-\infty}^{+\infty} L_{i} \cos (i M+\Pi) \\
\left(r_{1}\right) \sin \left(v_{1}+\Pi\right) \cdot 1 / 2 \sum_{i=-\infty}^{+\infty} Y^{(i)} \cos i M_{1}=1 / 2 \sum_{i=\infty}^{+\infty} L_{i} \sin \left(i M_{1}+\boldsymbol{I}\right) \\
1 / 2 z_{1} \sum_{i=-\infty}^{+\infty} Y^{(i)} \cos i M_{1}
\end{gathered}
$$

$$
L_{i}=1 / 2 a_{1} \sum_{k=-\infty}^{+\infty} Y^{(k-i)} \vartheta^{(k)}
$$

Schliesslich wird es noch nöthig, auf die Variabilität der $Y^{\prime}$ und $Z_{x, \lambda}^{(\sigma)}$ Rücksicht zu nehmen, und den Einfluss der Störungen $\gamma, \gamma_{1}$ der Radienvectoren zu berücksichtigen, was jedoch keinen wesentlichen Schwierigkeiten unterliegt.

Wien 1885 Juni 19. N. Herz.

\title{
Observations of (1) Ceres and (2) Pallas at the Opposition of 1885.
}

The accompanying positions of $(\mathrm{r})$ Ceres and (z) Pallas have been obtained by means of the filar-micrometer on the $4 \frac{1}{2}$ inch equatorial. The differential measures are corrected for refraction and the places of the planets for parallax. Proper motion has been applied to star 7 only.

\begin{tabular}{l|l|l|l|l|l|l|l|l}
\hline${ }_{1} 88_{5}$ & Winds.M.T. & $\Delta$ RA. & $\Delta$ NPD. & Cp. & RA. app. & NPD. app. & Red.ad l.app. & $*$ \\
\hline
\end{tabular}

(I) Ceres.

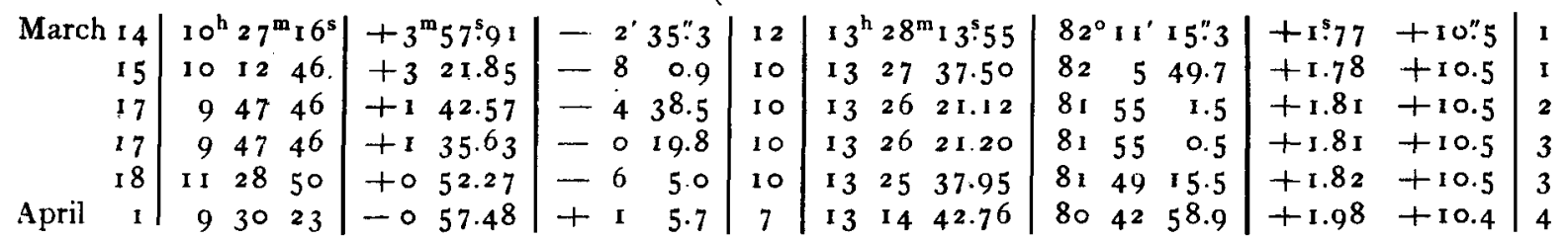

\begin{tabular}{|c|c|c|c|}
\hline * & RA. 1885.0 & NPD. I 885.0 & Authority \\
\hline $\mathbf{I}$ & I $3^{\mathrm{h}} 24^{\mathrm{m}}$ I $4^{\mathrm{s}}$ I I & $82^{\circ}$ I $3^{\prime} 3^{6 \prime \prime} 0$ & Yarn. 5570; Glasg. 3393 \\
\hline 2 & 132437.00 & $\begin{array}{lll}81 & 59 & 26.3\end{array}$ & Yarn. 5571 ; Glasg. 3395 \\
\hline 3 & I 32444.02 & 81556.6 & Glasg 3396 \\
\hline 4 & 131538.48 & $80 \quad 4 ! 39.3$ & Glasg. $335^{8}$ \\
\hline 5 & $12 \quad 17 \quad 14.74$ & $96 \quad 39 \quad 41.8$ & Lam $_{3} 1371$; Yarn. $5163 ;$ Brux. Cat. 1874.1074 . (Lam. NPD. rejected) \\
\hline 6 & $1215 \quad 56.88$ & 934447.0 & Lam $_{3}$ I $365 ;$ Glasg. $315^{2}$. (The NPD. differ by 4.7 ) \\
\hline 7 & $12 \quad 5 \quad 47.17$ & $8518 \quad 19.6$ & $\begin{array}{l}\text { Greenw. Cat. 1 840.62 1; Yarn. 5086; Schj. } 440 \text { I-2 ; Glasg. } 3 \text { I I } 7 \text {; } 9 \text { year I I I } 7 \text {; Brux. Cat. } \\
\qquad 1875.346 \text { and } 1876.725-6\end{array}$ \\
\hline 8 & I 5957.66 & $\begin{array}{lll}84 & \circ & 6.4\end{array}$ & Schj. $43^{64} ;$ Glasg. 3099 \\
\hline 9 & $\begin{array}{lll}12 & 2 & 10.02\end{array}$ & $\begin{array}{lll}83 & 19 & 26.0\end{array}$ & Glasg. 3103 \\
\hline
\end{tabular}

(2) Pallas.

\begin{tabular}{rr|rrr|rr|rr|r|r|rrr|rrr|r|r|r} 
Febr. 10 & 12 & 40 & 34 & +0 & 24.95 & + & 9 & 42.1 & 10 & 12 & 17 & 41.05 & 96 & 49 & 34.5 & +1.55 & +7.7 & 5 \\
20 & 9 & 50 & 36 & -0 & 16.80 & -5 & 31.8 & 13 & 12 & 15 & 41.46 & 93 & 39 & 28.6 & +1.72 & +9.9 & 6 \\
March 13 & 9 & 28 & 57 & -1 & 34.03 & + & 8 & 34.4 & 10 & 12 & 4 & 14.78 & 85 & 27 & 10.8 & +1.92 & +12.8 & 7 \\
17 & 10 & 49 & 59 & +1 & 25.39 & -12 & 0.0 & 10 & 12 & 1 & 24.84 & 83 & 48 & 23.8 & +1.94 & +13.1 & 8 \\
I8 & 10 & 33 & 39 & -1 & 29.32 & +4 & 58.1 & 10 & 12 & 0 & 42.48 & 83 & 24 & 41.4 & $+1.94+13.0$ & 9
\end{tabular}

Adopted mean Places of the Comparison Stars.

Observatory Windsor N. S. Wales I 885 April 29.

Fohn Tebbutt. 